\title{
DECIPHERING THE RELATIONSHIPS AMONG ENZYMATIC SYSTEMS AND VIRULENCE OF Beauveria bassiana: A REVIEW
}

\author{
Manish Dhawan ${ }^{1,2, *}$ (D), Neelam Joshi ${ }^{3}$, Samandeep Kaur ${ }^{1}$, Saroop Sandhu ${ }^{4}$, Meenu ${ }^{5}$ \\ ${ }^{1}$ Department of Microbiology, Punjab Agricultural University, Ludhiana- 141004, India \\ ${ }^{2}$ The Trafford Group of Colleges, Manchester-WA14 5PQ, United Kingdom \\ ${ }^{3}$ Department of Entomology, Punjab Agricultural University, Ludhiana- 141004, India \\ ${ }^{4}$ Department of Agronomy, University of Florida, Gainesville-FL 32611, United States of America \\ ${ }^{5}$ Department of Agriculture, Baba Farid College, Punjab-151001, India \\ Received - October 30, 2020; Revision - November 21, 2020; Accepted - December 24, 2020 \\ Available Online - December 30, 2020 \\ DOI: http://dx.doi.org/10.18006/2020.8(6).730.742
}

\section{KEYWORDS \\ Antioxidant machinery \\ Beauveria bassiana \\ Cuticle degrading enzymes \\ Entomopathogenic fungi \\ Pathogenesis \\ Virulence}

\begin{abstract}
Intensive crop production and extensive use of harmful synthetic chemical pesticides create numerous socio-economic problems worldwide. Therefore, sustainable solutions are needed for insect pest control, such as biological control agents. The fungal insect pathogen Beauveria bassiana has shown considerable potential as a biological control agent against a broad range of insects. The insight into the virulence mechanism of $B$. bassiana is essential to show the robustness of its use. B. bassiana has several determinants of virulence, including the production of cuticle-degrading enzymes (CDEs), such as proteases, chitinases, and lipases. CDEs are essential in the infection process as they hydrolyze the significant components of the insect's cuticle. Moreover, B. bassiana has evolved effective antioxidant mechanisms that include enzyme families that act as reactive oxygen species (ROS) scavengers, e.g., superoxide dismutases, catalases, peroxidases, and thioredoxins. In B. bassiana, the number of CDEs and antioxidant enzymes are characterized in recent years. These enzymes are believed to be crucial player of evolutionary process in this organism and their role in various mechanism of biological control. Recent discoveries have significantly increased our potential understanding on several potentially wanted unknown mechanisms of B. bassiana infection. This review focuses on the progress detailed in the studies of these enzymes and provides an overview of enzymatic activities and their contributions to virulence.
\end{abstract}

* Corresponding author

E-mail: dhawanmanish501@gmail.com (Manish Dhawan)

Peer review under responsibility of Journal of Experimental Biology and Agricultural Sciences.

Production and Hosting by Horizon Publisher India [HPI] (http://www.horizonpublisherindia.in/).

All rights reserved.
All the articles published by Journal of Experimental Biology and Agricultural Sciences are licensed under a Creative Commons Attribution-NonCommercial 4.0 International License Based on a work at www.jebas.org.

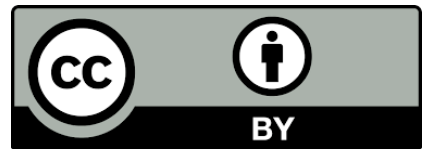




\section{Introduction}

Insect pests cause severe damage to crops, and an 18 to $20 \%$ reduction of the annual global crop yield is recorded by pests in the last decade. (Oerke \& Dehne,2004). Eight percent loss of crops yield was estimated in Brazil while with the application of chemical control measures (Oliveira et al., 2014). The application of chemical pesticides to control insect pests plays an inevitable role in increasing agricultural production and ensures the quality of supplied food (Meenu et al., 2020). Chemical pesticides have been widely applied in agriculture for several decades. Environmental and health risks associated with pesticides are rising concerns for the applications of chemical insecticides to reduce large-scale insect pest infestations and have led to renewed interest in the development of microbial agents as an alternative control of crop pests (Oliveira et al., 2014; Joshi et al.,2019; Litwin et al., 2020). Various microbial agents, such as Bacillus thuringiensis, Beauveria bassiana, Verticillium lecanii, and Metarhizium anisopliae, are currently used as biological control agents for different insect pests to reduce the crop loss and spread of various arthropod-borne diseases (Alavo, 2015; Dhawan et al., 2018; Kaur et al., 2017; Carolino et al., 2019).

The cosmopolitan anamorphic fungus, Beauveria bassiana ( $B$. bassiana)(Balsamo) Vuillemin (Ascomycota: Hypocreales) is a well-recognized and most exploited biological control agent (BCA). B. bassiana is known to infect hundreds of host species belonging to most insect orders, and it is the most appropriate BCA in temperate agriculture regions (Zimmerman, 2007; Glare et al., 2008; Singh \& Joshi 2020). The success of B. bassiana as biopesticide not only depends on its high efficacy against insect pests but also on its non-pathogenic behavior towards non-target pests (Thungrabeab \& Tongma 2007; Bhadauria et al., 2013).

Unlike bacteria or viruses, $B$. bassiana infect insects by direct penetration through cuticle. However, insect cuticle serves as a physical barrier against fungal attack; it is a structure formed by crystalline chitin nanofibers inside proteins, polyphenols, and lipids matrix (Vincent \& Wegst 2004; Sharma et al., 2017). So, B. bassiana secretes several extracellular cuticle degrading enzymes (CDEs) such as proteases, chitinases, and lipases to degrade the major constituents of the insect's cuticle and allow hyphal penetration into the insect's cuticle (Dhawan \& Joshi 2017) (Figure 1). CDEs play pivotal roles in the infection mechanism and are considered as essential virulence governing factors of $B$. bassiana. CDEs enable $B$. bassiana to coexist with the changing metabolic processes associated with the host's disease (Wang et al. 2005; Cho et al. 2006). In addition to CDEs, B. bassiana has evolved several stressrelated enzymes such as superoxide dismutases (SODs), catalases, and thioredoxins (TRXs). Previously, these enzymes are known to

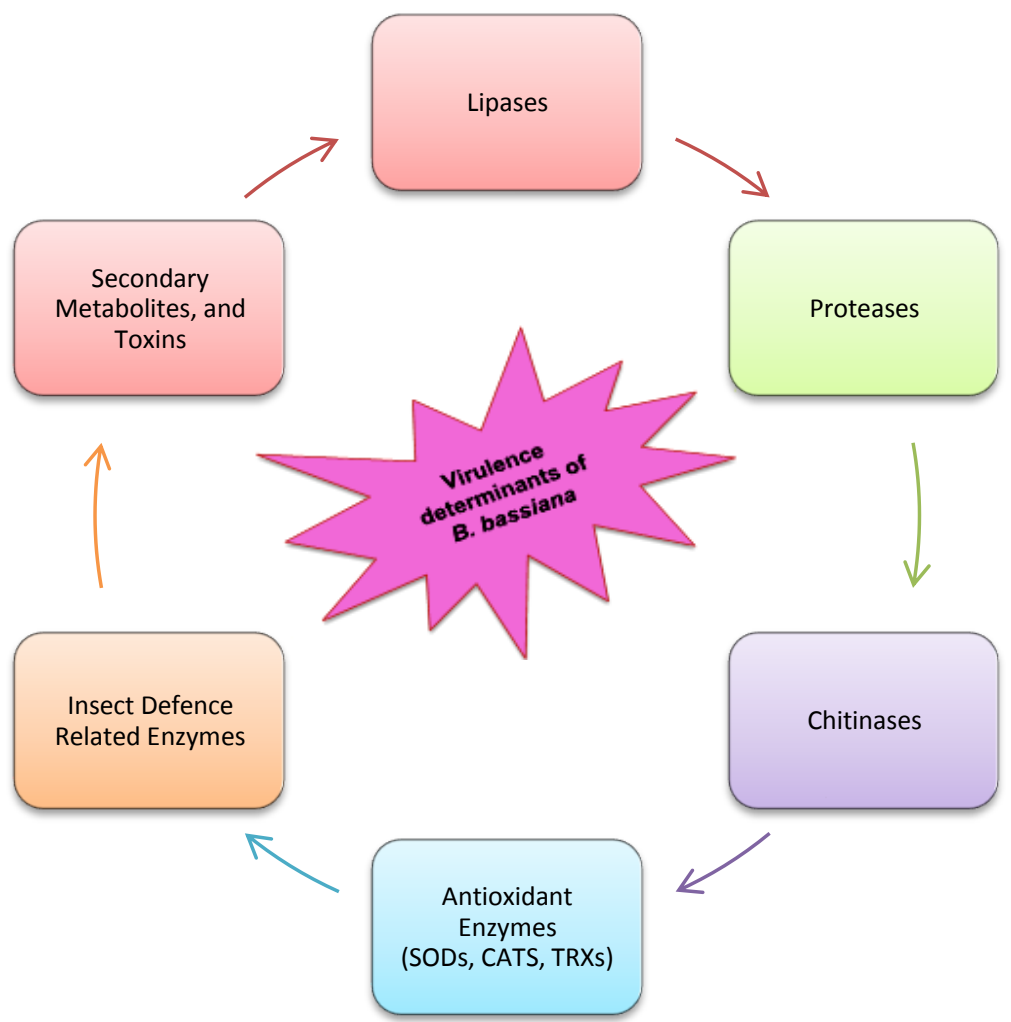

Figure 1 Virulence governing factors of $B$. bassiana and the proposed sequence of action during the infection process. 
combat abiotic stresses like osmotic stress, ultraviolet (U.V.) radiations, high/low salt, and high/low temperature associated stress. However, their role in the virulence of $B$. bassiana is still unclear. Antioxidant enzymes determine the shelf life of commercially available $B$. bassiana and determine its efficacy in field applications (Kaur \& Joshi 2014; Wang et al., 2020). Moreover, B. bassiana produces several secondary metabolites and enzymes such as benzoquinone reductase and ribotoxins, which provide protection against the insects' immune response and help to kill its target pest.

However, it is still a mystery about how all these enzymes work together and determine the virulence of B. bassiana? The sequence of action of CDEs is still unclear, and this review explores the roles of CDEs at various steps of the infection in insect pests. The deep insights into the enzymatic machinery of B. bassiana will lead us to a better biocontrol approach and sustainable solution to control arthropod pests. Hence, the present review focuses on the roles of the various CDEs and stress-related enzymes in the pathogenesis of B. bassiana.

\section{Extracellular Cuticle Degrading Enzymes (CDEs)}

\subsection{Lipases}

Lipases play fundamental role in cellular lipid metabolism and participate in the storage, processing, digestion, and transport of lipid substrates (Gupta et al., 2015). These enzymes hydrolyze the ester bonds of triacylglycerols, which leads to the release of free fatty acids, diacylglycerols, monoacylglycerols, and glycerol (Keyhani, 2017). Lipases play significant roles in the host infection process as these enzymes are essential in initiating the infection process. Lipid degradation in the insect cuticle's outer layer has been associated as a growth phase before fungus penetration, leading to the initiation of the infection process. It was also suggested the subsequent synthesis of hydrolytic lipases, among other enzymes, degrade the fatty acids in the cuticle and release nutrients to the fungus (Beys da Silva et al., 2010).

Moreover, lipases support the adhesion of germinating spores to insect cuticles by increasing hydrophobic interactions between the fungus and the cuticle surface which helps in initiating the infection process (Santi et al., 2010). Further, Khan et al. (2012) correlated the toxicity of B. bassiana with the enzymatic activities, and proposed that the lipases participate more in total virulence or pathogenicity as compared to protease and chitinase while protease was assumed to participate more than chitinase. They evaluated the pathogenicity of B. bassiana isolates against Myzus persicae and reported variability in the mortality against the target pest and virulent isolates showed marked enzymatic activities as compared to less virulent isolates.
Differential secretome of $B$. bassiana induced by the Rhipicephalus microplus cuticle was evaluated by the multidimensional protein identification technology. Fifty proteins from a total of 236 proteins were identified exclusively in infection condition, assigned to different aspects of infection such as host adhesion, cuticle penetration and fungal defense, and stress. Several bioinformatic analyses suggested that the highest number of proteins in the differential secretome possesses lipase activity (Santi et al., 2018).

B. bassiana contains a broad suite of CYP P450 genes (approximately 83) that act on a wide range of compounds, and only a subset of these enzymes acts on the hydrocarbons (Urlacher et al. 2004). Series of enzymes are required for the various hydrocarbon substrates found on most insect cuticles (Keyhani, 2017). The substrate specificity and role of one B. bassiana hydrocarbon-acting hydroxylase CYP P450 has been characterized named as Bbcyp52xl gene (Zhang et al., 2012). Purified BbCYP52X1 was shown to display terminal hydroxylation activity against midrange fatty acids (C12:0 and C14:0) and epoxy stearic acid. Whereas, it showed negligible activity against long chain fatty acids. Loss of function of B. bassiana mutants (Bbcyp52x1) resulted in reduced virulence against Galleria mellonella larvae (Table 1). These mutants showed decreased germination on grasshopper wings as compared to the wild type parent and complemented controls.

In contrast to this, individual targeted gene knockouts of five other (putative) hydrocarbon-specific $B$. bassiana CYP P450s (CYP5337A1, CYP52G11, CYP617N1, CYP53A26, and CYP655C1) did not show any significant decrease in virulence and any significant defects in lipid utilization (Pedrini et al. 2013). Zhou et al. (2018) reported differential expression of lipases encoding genes in B. bassiana infecting two different hosts. Cytochrome P450 CYP68N1 was up-regulated to implicate in insect hydrocarbon degradation in B. bassiana infecting Galleria mellonella, while Cytochrome P450 CYP625A1 was up-regulated in B. bassiana infecting Plutella xylostella. These studies suggest the differential secretion of lipases against different insect pests. In the future, there is a need for more studies to establish the exact roles of CYP P450 proteins in the virulence of B. bassiana.

\subsection{Proteases}

Proteases constitute a large group of hydrolytic enzymes that cleave the peptide bonds of proteins and break polypeptide protein structures into small peptides and amino acids (Sharma et al. 2006). The production of extracellular proteases is a crucial factor in determining the virulence of $B$. bassiana against the target host. It is believed that after the epicuticle has been broken down by lipases, the invading fungi produces sufficient quantities of Pr1 (serineprotease) that successfully degrade the proteinaceous material. The 
Table 1 Enzymes and their encoding genes involved in pathogenesis and physiological processes of B. bassiana.

\begin{tabular}{|c|c|c|c|}
\hline Enzyme & Gene name & Knock-out mutant phenotype & Reference \\
\hline Lipase/Esterase & $\begin{array}{l}\text { Cyp p } 450 s \\
\text { Bbcyp } 52 x 1\end{array}$ & $\begin{array}{c}\text { N.A. }{ }^{*} \\
\text { Reduced virulence, germination and cuticle breaching }\end{array}$ & $\begin{array}{l}\text { Pedrini et al. (2013) } \\
\text { Zhang et al. (2012) }\end{array}$ \\
\hline Protease & $\begin{array}{l}\text { Cdep1 } \\
\operatorname{Pr} 1, \operatorname{Pr} 2\end{array}$ & $\begin{array}{l}\text { N.A.* } \\
\text { N.A.* }\end{array}$ & $\begin{array}{c}\text { Zhang et al. (2008) } \\
\text { Joshi et al. (1995), Dias et al. } \\
\text { (2008) }\end{array}$ \\
\hline Chitinase & $\begin{array}{c}\text { Bbchit1 } \\
\text { Chi1, Chi2, ChsA2 }\end{array}$ & $\begin{array}{c}\text { Overexpression increased infection efficiency } \\
\text { N.A. }\end{array}$ & $\begin{array}{c}\text { Pinnamaneni et al., } 2010 \\
\text { Liu et al., } 2013\end{array}$ \\
\hline Catalases & CatA-D, CatP & Reduced stress tolerance and decreased virulence & Wang et al., 2013 \\
\hline $\begin{array}{c}\text { Superoxide } \\
\text { dismutases (SODs) }\end{array}$ & Sod1-Sod5 & Reduced stress tolerance and slight decreased virulence & $\begin{array}{l}\text { Xie et al. (2012); Xie et al. (2010); } \\
\text { Li et al. (2015) }\end{array}$ \\
\hline Thioredoxins (TRXs) & $\operatorname{Tr} x 1-\operatorname{Tr} x 6$ & $\begin{array}{c}\text { Reduced abiotic stress tolerance, virulence. Decreased } \\
\text { germination and conidiation }\end{array}$ & Zhang et al. (2015) \\
\hline
\end{tabular}

${ }^{*}$ N.A. (Not Available)

most frequently studied proteolytic enzymes are the subtilisin-like serine-protease $\operatorname{Pr} 1$ and trypsin-like protease $\operatorname{Pr} 2$. The $\operatorname{Pr} 1$ gene is related to eleven isoforms that have been identified and cloned, including a metalloprotease (St. Leger et al., 1994).

According to Dhar \& Kaur (2010) reported that the protease is one of the most important and the earliest enzyme known to be involved in host invasion. Seventeen isolates of $B$. bassiana were evaluated for extracellular protease production, and the highest protease activity was observed during four to six days of culture incubation. Subtilisin type $\operatorname{Pr} 1$ and trypsin type $\operatorname{Pr} 2$ activities were measured using different media. Minimal medium supplemented with $1 \%$ casein showed high protease production, whereas minimal medium supplemented with 2 percent colloidal chitin could also induce Pr1 activity. They further reported that $66 \mathrm{KDa}$ protease was commonly observed in different isolates of $B$. bassiana.

In the process of enzymatic degradation, the type of chymoelastase serine protease Pr1 served as a crucial cuticledegrading enzyme because $70 \%$ of the cuticle was composed of protein Pr1. Subtilisin type Pr1 has been reported to show a considerable ability to degrade the insect cuticle with a high concentration at the site of the penetration peg or the germ tube (St. Leger et al. 1996). The molecular structure of subtilisinlike protease $\operatorname{Pr} 1$ consists of five cysteines forming two disulfide bridges, and the residual cysteine found near the catalytic site consists of Asp39, His69, and Ser224.

Liu et al. (2007) also determined the activities of $\operatorname{Pr} 1$ and $\operatorname{Pr} 2$ enzymes. They found that proteases are secreted during the first cuticle degradation stage, and they stimulate the signal transduction mechanism by activating protein kinase A (PKA) mediated by cyclic adenosine $3^{\prime}, 5^{\prime}$-monophosphate (cAMP). It validated that the infection of the cuticle initializes the extracellular involvement of protease Pr1 in cuticle penetration.
Additionally, protease Pr1 also found as a virulence indicator in several entomopathogenic fungi (EPF) apart from B. bassiana (Castellanos-Moguel et al., 2007).

Thakur et al. (2005) identified three different proteases (isoenzymes) in different $B$. bassiana isolates by protease zymography. They reported the variability in the protease activity among the different strains of B. bassiana. The variability in the secretion of proteases leads to differences in the virulence of fungal isolates. Recently, Mancillas-Paredes et al. (2019) compared the production of protease in inducing and non-inducing medium and reported the significantly higher production of protease in induction medium and suggested the positive correlation of protease with the virulence of B. bassiana against Zabrotes subfasciatus.

New strategies such as genetic engineering and recombination of proteases genes have improved the virulence of EPF (Fang et al. 2009). Genetic modification of B. bassiana has done through the co-transformation of scorpion aaIT and Metarhizium prlA genes. The binary transformants did not show improved insecticidal activity as compared to the aaIT single transgenic isolates. Hence, it was evident that the protease PR1A can degrade AAIT when both are present in culture media or insect hemolymph, and provided a reasonable explanation for their non-synergistic effect. These studies conclude that the virulence enhancement seen in binary transformants is mainly due to the secretion of PR1A, which could accelerate cuticle penetration and activate hemolymph prophenol oxidase to melanize the insect body (St. Leger et al. 1992).

In another study, cDNA of the protease gene $C D E P 2$ isolated from B. bassiana. It is combined with the crylAc gene of Bacillus thuringiensis, while the expression of the gene was regulated by the native promoter of the fungus. This 
experiment improved the insecticidal activity of EPF against the Helicoverpa armigera larvae (Xia et al. 2009). Overexpression of protease increases the virulence of fungal isolate against the target pest. Hence, proteases can be considered as an essential factor that governs the virulence of B. bassiana (Zhang et al. 2008; Fan et al. 2010). In future, protease production can be enhanced by genetic modifications to increase the virulence of EPF against the target insect pest.

\subsection{Chitinases}

Insect's cuticle is primarily composed of chitin, an unbranched polymer of $\mathrm{N}$-acetyl glucosamine monomers that are linked by $\beta-1,4$ glycosidic bonds and chitinases catalyze the hydrolysis of the $\beta-1,4$ linkages and resulting in the release of short-chain chitooligomers or monomers. Chitinases are members of glycoside hydrolase (G.H.) families 18 \& 19 and widely distributed in fungi, insects, and vertebrates, and do not share similar sequences $(\mathrm{Li}, 2006)$.

Recent studies have shown that $B$. bassiana produces an extensive amount of endo-chitinases and exo-chitinases, and their activities are positively correlated with the virulence against the insect pests (Sanchez-Perez et al. 2016; Dhawan \& Joshi 2017). Endochitinases hydrolyze the $\beta-1,4$-glycosidic bond inside the chitin molecule and exo-chitinases hydrolyze $\mathrm{N}$-acetylglucosamine oligomers formed during the action of endo-chitinases. The combined action of endo- and exo-chitinases is required for the complete degradation of insect chitin during the infection process (Oyeleye \& Normi 2018; Hamid et al., 2013).

Several findings state a positive correlation between virulence and chitinase activities of $B$. bassiana. Kim et al. (2010) reported that the higher levels of extracellular chitinases in B. bassiana are responsible for virulence towards the aphid Aphis gossypii. MatiasMontesinos et al. (2011) found that a high virulence of B. bassiana mutant was associated with increased production of chitinase. The virulence of the mutant $B$. bassiana was significantly higher from the wild type. Pelizza et al. (2012) also reported that the fungal isolates of B. bassiana with the highest levels of chitinase activities were more pathogenic against Tropidacris collaris.

Similarly, Firouzbakht et al. (2015) compared two different isolates of B. bassiana against Andrallus spinidens and reported that the chitinase activity is significantly higher in the virulent isolate with lesser LC50 value as compared to less virulent isolate of B. bassiana. Perinotto et al. (2014) found that the most virulent strain of EPF expressed the highest chitinase activity against Rhipicephalus microplus. Recently, Alali et al. (2019) isolated thermotolerant strains of B. bassiana and reported that the strains with the lowest LD50 and LT50 values possessed significantly higher values of chitinase activity. This suggests a positive correlation of chitinase activity with the pathogenicity and virulence of B. bassiana.
B. bassiana expresses 20 different chitinases divided into three subgroups: eight appertaining to subgroup A without a chitinbinding domain (ChBD); four appertaining to subgroup B (one $\mathrm{ChBD}$ in the extreme $\mathrm{C}$ terminal) and eight appertaining to subgroup C having two ChBD sites (Xie et al., 2012). Interestingly, the two endogenous Beauveria chitinases (chitl and chit2) appear to respond to host cuticles do not contain ChBD often seen in similar enzymes.

Fang et al. (2005) reported that the overproduction of Bbchitl enhanced the virulence of B. bassiana against Myzus persicae, as indicated by a significant reduction in LC50 and LT50 of the transformants compared to the values for the wild-type strain. In another study, the virulence of $B$. bassiana improved against silkworm moth Bombyx mori with chitinase production from a recombinant Bbchitl gene. The recombinant was constructed by fusing the Bbchitl gene with the ChBD under the regulation of the promoter. The overexpression of chitinase leads to significant changes in the virulence (Fan et al. 2007). Hence, the constitutive expression of a mutated chit1 gene containing a chitin-binding domain proved to be a better approach for creating virulent strains of B. bassiana. Moreover, Pinnamaneni et al. (2010) also found that the genetically modified form of $B$. bassiana is more effective in expressing pathogenicity as the exochitinase activity was maximum compared to that of crude extract (Table 1). Hence, the production of extracellular chitinases is an essential factor in governing the virulence of $B$. bassiana.

\section{Antioxidant enzymes}

In response to fungal attack, insect pest also produces free radicals or reactive oxygen species (ROS) that can cause cell damage by oxidizing cell components, such as DNA, protein, and lipids (Figure 2).Survival of B. bassiana relies upon antioxidant defense systems to scavenge ROS (Zhang \& Feng, 2018). Upon field application, formulated fungal cells are inevitably exposed to certain abiotic stresses, such as persistently high temperatures close to or above their upper thermal limits, UV irradiation, and agrochemicals applied for controls of plant diseases and weeds (Wang et al., 2020). Such exposures during field applications may reduce the efficacy of fungal pesticides applied for insect pest control, making it necessary to understand the roles of antioxidant enzymes and molecular mechanisms involved in, and hence explore effective means to improving, multiple stress tolerance of formulated fungal cells (Tong \& Feng, 2019; Ying \& Feng, 2019; Tong \& Feng, 2020). Such systems consist mainly of superoxide dismutases (SODs), catalases, and thioredoxins (TRXs) (Kwok et al. 2004; Kaur et al. 2016; Kaur et al. 2017; Singh et al., 2020). A suite of SODs, catalases, and TRXs proteins have been investigated in B. bassiana. These enzymes play several important physiological roles and help in the pathogenesis of $B$. bassiana (Zhang \& Feng, 2018). 


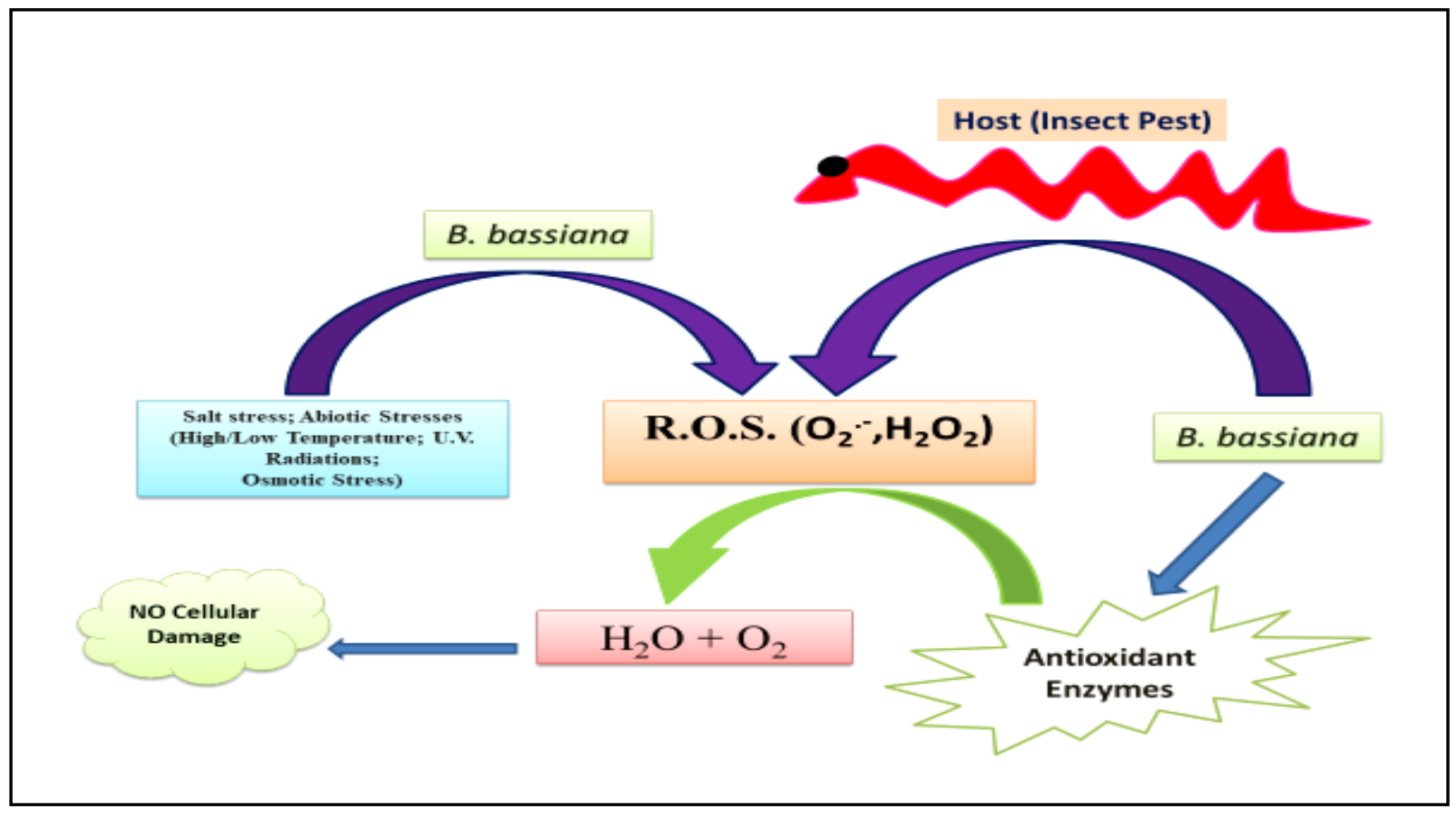

Figure 2 The antioxidant machinery provides protection against reactive oxygen species (ROS) generated from abiotic stresses and host (insect pest) immune response.

\subsection{Superoxide dismutases (SODs)}

The SOD family is the first line of protection against the oxidative stress from superoxide radical anions and comprises three types of isoforms that are co-factored with $\mathrm{Cu}^{2+} \mathrm{Zu}^{2+}, \mathrm{Mn}^{2+}$, and $\mathrm{Fe}^{2+}$, respectively (Culotta et al., 2006) (Figure 3). SODs catalyze the conversion of superoxide radical anions into peroxide, and five different B. bassiana SODs (Sod1-5) have been characterized, including cytosolic $\mathrm{Cu} / \mathrm{ZnSOD}$ (Sod1) and MnSOD (Sod2), mitochondrial MnSOD (Sod3) and FeSOD (Sod4), and cell-wall anchored Cu/ZuSOD (Sod5) (Xie et al., 2012; Li et al., 2015)(Figure 3). Several SODs found in B. bassiana have been explored as a crucial factors for its potential biological control and tolerance to environmental stresses like high temperature, solar U.V. irradiation (Yao et al., 2010), and fungicidal application (Song et al. 2012). Inactivation of the Sod genes through RNA interference (RNAi) knockdown resulted in decreased tolerance to peroxide, oxidative stress, and UVA/B exposure. However, a small decrease in virulence has been observed for the mutants during insect bioassays ( $\mathrm{Li}$ et al., 2015). Moreover, a cytosolic manganese-cored SOD (Bbsod2) overexpressed in B. bassiana led to significant increases in virulence against target insect pests (Xie et al., 2010).

\subsection{Catalases}

Catalases (CATs) are the enzymes that catalyze the conversion of peroxide to oxygen and water (Figure 3). Five members in the catalase family of B. bassiana were functionally characterized by one-by-one gene knockout, followed by enzymatic, transcriptional and phenotypic analyses (Wang et al., 2013). These five different $B$. bassiana CAT genes include CatA (spore specific enzyme), CatB (secreted), CatC, (cytoplasmic), CatD (secreted or peroxisomal), and CatP (peroxisomal) (Figure 3). These catalases have been reported to contribute to stress response and virulence of B. bassiana (Wang et al. 2013). A peroxisomal catalase in B. bassiana was clued as an enzyme associated with insect hydrocarbon catabolism due to the increase of its activity by the replacement of glucose with an insect-like hydrocarbon in medium (Pedrini et al., 2006). The overexpression of a catalase in Metarhizium anisopliae facilitate pathogenesis by reducing conidial germination time and enhance the fungal virulence against Plutella xylostella larvae (Hernandez et al., 2010). These studies indicate that, like SODs, catalases are involved in mediating not only fungal growth and development but also stress tolerance and virulence to determine the biocontrol potential of B. bassiana.

Chantasingh et al. (2013) identified a set of differentially expressed genes in B. bassiana in response to Spodoptera exigua larvae. Polymerase chain reaction-suppression subtractive hybridization (PCR-SSH) was used by which they identified differentially expressed genes during the initial aspects of the fungal-insect interaction. Ten fungal genes were identified by PCR-SSH, and these were further confirmed to be up-regulated 
by semiquantitative RT-PCR. They further characterized the catalase gene (catE7), which is implicated in stress resistance and has a role in the pathogenesis of $B$. bassiana. They constructed a transgenic strain of $B$. bassiana. This strain was overexpressing CatE7, and fungal transformant lines with extra catE7 copies (Bb::BbcatE7) showed two-fold higher catalase activity than the wild type Bb::BbcatE7 strains. These strains were found to be germinated faster than the wild-type parent and exhibited significantly higher virulence against $S$. exigua larvae. They suggested that responses mediated by catalases play an important role in the fungal-insect infection process, and manipulation of catalase expression can produce more virulent fungal strains for efficient insect control.

\subsection{Thioredoxins (TRXs)}

Thioredoxins and thioredoxin reductases (TRXs-TRRs) are small molecular weight $(12 \mathrm{kDa})$ oxidoreductase enzymes that help in maintaining the redox balance of the cell. The TRX-TRR system requires NADPH for its activity. A typical TRX bears two redoxactive cysteine residues $\left[\operatorname{Trx}-(\mathrm{SH})_{2}\right]$ in the motif of its conserved active site (WCGPC) essential for the redox of sulfide and disulfide (Mahmood et al., 2013). Reduced TRX (Trx-SH) can reduce cysteine residues in proteins and becomes oxidized type (Trx-S2) after the reduction is completed; the TRX redox required for the recycling of $\operatorname{Trx}-\mathrm{S}_{2}$ and $\operatorname{Trx}-\mathrm{SH}$ is catalyzed by
TRR, which employs NADH as an electron donor through a central NADPH-binding domain (Arner \& Holmgren 2000; Mustacich \& Powis 2000).

TRXs respond to ROS to regulate a wide range of signaling and developmental processes. They act as potent antioxidants by catalyzing protein reduction via cysteine thiol-disulfide exchange and are essential in many organisms. Six B. bassiana TRXs have been named TRXs 1 to 6 , and TRX 1-4 located in the cytoplasm of the fungal cells. Whereas TRX5 located in the nuclear membrane, and TRX6 present in the mitochondrial membrane (Zhang et al., 2015). Targeted gene knockouts of any of the TRX genes resulted in varying degrees of reduced virulence. Moreover, in B. bassiana, two TRRs (Trr1/2) are present in the cytoplasm, and Trr1 takes part in the positive regulation of Peroxidases (PODs) and SODs activities, which were significantly decreased in $\Delta \operatorname{trr} 1$ mutants. At the same time, Trr2 does not play significant roles (Zhang et al., 2016a).

In recent years dozens of antioxidant enzymes in the SOD, CAT, and TRX families have been characterized for their activities to catalyze different types of ROS and contributions to cellular response to oxidative stress in B. bassiana. Many of these enzymes are not essential for fungal viability because loss-of-function mutations can be constructed, although, in many mutants, phenotypes could be entirely or partially compensated by upregulated expression of certain partner

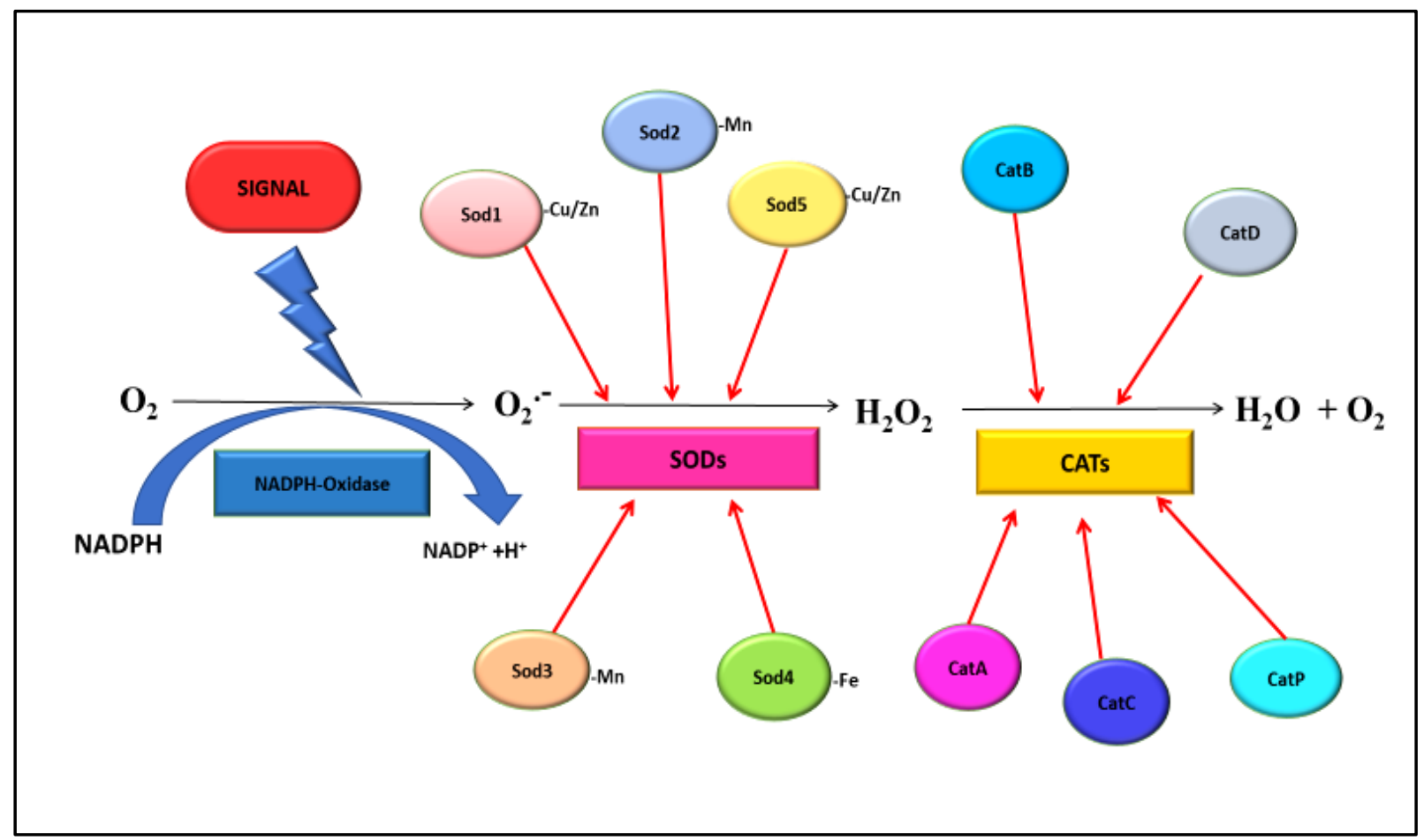

Figure 3 The constituents of antioxidant machinery and its functioning in B. bassiana. NADPH Oxidase (NOX) generates superoxide radical anions under various conditions and superoxide dismutases (SODs) decompose superoxide radical anions into hydrogen peroxide $\left(\mathrm{H}_{2} \mathrm{O}_{2}\right)$, which is further decomposed intooxygen and water by catalases (CATs).

Journal of Experimental Biology and Agricultural Sciences http://www.jebas.org 
genes from the same family. Exceptions include Sod4 (FeSOD), which appear to be indispensable for the fungal viability due to failures to obtain deletion mutants despite many attempts (Li et al., 2015; Zhang et al., 2016b). Apart from the complementary roles of partners within each family of antioxidant enzymes, profiled transcripts and quantified enzyme activities illustrate inter-family interactions at transcriptional and translational levels, implying that various enzymes of the antioxidant machinery are well coordinated to protect $B$. bassiana from abiotic stresses and insect response (Figure 3).

\section{Insect defense-related enzymes}

The nutrient and water limiting insect epicuticle, when coupled with the secretion of antifungal compounds, act as a potential defense against the microbial attack (Ortiz-Urquiza \& Keyhani, 2014). It has long been recognized that certain insect species are resistant to infection by $B$. bassiana, even though other closely related species are susceptible. Tribolium castaneum (the red flour beetle) is one such resistant insect species and produces large amounts of benzoquinones that act as a barrier and provide protection against microbial attacks. For example, $T$. castaneum express a strong resistance against the several strains of $B$. bassiana. Some wild-type strains of the B. bassiana result recorded only $20-25 \%$ mortality when tested against $T$. castaneum. However, B. bassiana produces a benzoquinone reductase (BqrA) that can neutralize the effect of benzoquinones, and targeted gene knockouts of the BqrA gene reduced mortality to $10 \%$. A strain overexpressing BqrA showed higher virulence that ranged from 40 to $45 \%$ (Pedrini et al., 2015). Apart from benzoquinone reductase, several reports suggest the role of secondary metabolites and toxins such as oosporein, destruxins, cylosporines, beauvericin, tenellin, and brassinolide in protecting B. bassiana against insect's resistance and immune responses (Ortiz-Urquiza et al., 2013; Gibson et al., 2014). Zibaee et al. (2011) suggested that the secondary metabolites produced by $B$. bassiana disable several immune mechanisms of Eurygaster integriceps, thus helping the fungus to overcome and kill its host. Fan et al. (2013) found that $B$. brongniartii produces secondary metabolites that disable the immune mechanisms of Dendrolimus tabulaeformis, allowing the fungus to overcome and then kill its host.

A recent study proposes, an unusual virulence factor, ribotoxin (Rib), secreted by B. bassiana, could repress insect host immune responses to facilitate microbial infections. Ribotoxins are extracellular secreted ribonucleases (RNases), including ribotoxic cytotoxicity and insecticidal activity. Inset bioassays demonstrated that the Rib gene contributes to fungal virulence; $\Delta R i b$ mutant conidia injection killed insect larvae after a time delay, and ROS response levels inside $\Delta R i b$ mutant-infected silkworm hemocytes were much more robust. These differences were most probably due to the absence of the Rib gene, which resulted in an enhancement of host innate immune responses throughout fungal infection. Consequently, most conidia of the $\Delta R i b$ mutants were likely killed or eliminated by much stronger ROS niches around the phagocytosed hemocytes, and higher concentrations of AMPs were secreted into the hemocoel by fat bodies. This implies that ribonuclease is a crucial virulence factor that provides protection against insect immune response to fungal cells (Yuan et al., 2020).

\section{Conclusion and future perspectives}

The CDEs such as lipases, chitinases, and proteases, and antioxidant enzymes, including SODs, Catalases, TRXs, are significant components of the enzymatic machinery that governs virulence in EPF such as B. bassiana. CDEs play a significant role in degrading the insect's cuticle. In contrast, antioxidant enzymes help in several physiological processes and protect infective units, i.e., conidia of EPF under stress conditions. The efficiency of enzymatic machinery determines the fungal isolates' virulence, which can differ among the different isolates of $B$. bassiana. Several comprehensive studies have been conducted to investigate the enzymatic machinery of $B$. bassiana, and multiple members in most of these enzyme families are functionally explained in B. bassiana. However, only a very few have been explored in other EPF, such as Metarhizium anisopliae, Lecanicillium lecanii, and Isaria fumosorosea that are also proclaimed as potential microbial agents to manage several agriculturally important insect pests. It is much needed to investigate the enzymatic mechanisms in other groups of EPF for more reliable biocontrol approaches.

In future, the relationship of enzymatic activities with virulence can be exploited as a useful tool to select the most virulent fungal isolates of B. bassiana or any EPF against target insect pest. This knowledge will improve biocontrol products' efficiency, which can serve as alternatives to chemical pesticides to prevent crop loss. Moreover, once specific virulence factors are identified, it will be essential to explore the natural variation (in nucleotide sequence and expression of these genes) in the fungal population. It is also highly relevant to anticipate how insects can undergo adaptation to evade the action of fungal virulence factors and thereby develop resistance to the biocontrol agent. Further, CDEs and antioxidant enzymes can be genetically manipulated to create more virulent strains of $B$. bassiana. However, the use of genetically modified organisms has numerous complications that reduce their utilization in biological control.

\section{Ethics approval and consent to participate:}

\section{Not applicable}

\section{Consent for publication}

All the authors have agreed for publication. 


\section{Competing interests}

The authors declare that they have no competing interests.

\section{Funding}

No Funding.

\section{Author's contribution}

MD and NJ both conceptualized the idea and wrote the review article along with the valuable inputs from all the other authors. All authors read and approved the final manuscript.

\section{Acknowledgements}

All authors acknowledge Dr. Manisha Parmar, Department of Microbiology, Punjab Agricultural University, Ludhiana, India, for her valuable suggestions and help.

\section{References}

Alali S, Mereghetti V, Faoro F, Bocchi S, Al Azmeh F, Montagna M (2019)Thermotolerant isolates of Beauveria bassiana as potential control agent of insect pest in subtropical climates. PLoS ONE14(2): e0211457https://doi.org/10.1371/journal.pone.0211457.

Arner ES, Holmgren A (2000) Physiological functions of thioredoxin and thioredoxin reductase. European Journal of Biochemistry267: 6102-6109.

Alavo TB (2015) The insect pathogenic fungus Verticillium lecanii (Zimm.) Viegas and its use for pests control: A Review. Journal of Experimental Biology and Agricultural Sciences 3(4): 337-345.

Bhadauria BP, Singh PK, Shailesh P, Zaidi NW, Singh US (2013) Characterization and biocontrol potential of entomopathogenic fungus, Beauveria bassiana isolates against Spilarctiaobliqua. Journal of Environmental Biology 34(5): 917-21.

Beys da silva W, Santi L, Schrank A, Vainstein MH (2010) Metarhizium anisopliae lipolytic activity plays a pivotal role in Rhipi cephalus (Boophilus) microplus infection. Fungal Biology 114:10-15.

Carolino AT, Gomes SA, Teodoro TBP, Mattoso TC, Samuels RI (2019) Aedesaegypti pupae are highly susceptible to infection by Metarhizium anisopliae blastospores. Journal of Pure and Applied Microbiology 13(3):

https://doi.org/10.22207/JPAM.13.3.36

Castellanos-Moguel J, Gonzalez-Barajas M, Mier T, Reyes Montes MR, Aranda E, Toriello C (2007) Virulence testing and extracellular subtilisin-like (Pr1) and trypsin-like (Pr2) activity during propagule production of Paecilomyces fumosoroseus isolates from whiteflies (Homoptera: Aeyrodidae). Revista Iberoamericana de Micología 24: 62-68.

Chantasingh D, Kitikhun S, Keyhani NO, Thoetkiattikul KBH, Pootanakit K, Eurwilaichitr L (2013) Identification of catalase as an early up-regulated gene in Beauveria bassiana and its role in entomopathogenic fungal virulence. Biological Control 67: 85-93.

Cho EM, Boucias D, Keyhani NO (2006) EST analysis of cDNA libraries from the entomopathogenic fungus Beauveria (Cordyceps) bassiana. II. Fungal cells sporulating on chitin and producing oosporein. Microbiology152: 2855-64.

Culotta VC, Yang M, O'Halloran TV (2006) Activation of superoxide dismutases: putting the metal to the pedal. Biochimica et Biophysica Acta (BBA) - Molecular Cell Research1763: 747-758.

Dhar P, Kaur G (2010) Production of cuticle degrading proteases by Beauveriabassianaand their induction in different media. African Journal of Biochemistry Research 4(3): 65-72.

Dhawan M, Joshi N (2017) Enzymatic comparison and mortality of Beauveria bassiana against cabbage caterpillar Pieris brassicae LINN. Brazilian Journal of Microbiology 48 (3): 522-529.

Dhawan M, Kaur S, Chopra H, Kalra G, Kaur S, Sharma M, Khosla R (2018) Evaluation of cost-effective methodology for the isolation of bacillus thuringiensis and its toxin production. Research Journal of Life Sciences, Bioinformatics, Pharmaceutical and Chemical Sciences 4(3): 464-478. https://doi.org/10.26479/2018.0403.41

Dias B, Neves P, Furlaneto-Maia L, Furlaneto MC (2008) Cuticledegrading proteases produced by the entomopathogenic fungus Beauveria bassiana in the presence of coffee berry borer cuticle. Brazilian Journal of Microbiology 39: 301-306.

Fan J, Xie Y, Xue J, Liu R (2013) The effect of Beauveria brongniartii and its secondary metabolites on the detoxification enzymes of the pine caterpillar, Dendrolimus tabulaeformis. Journal of Insect Science 13: 44.

Fan YH, Fang WG, Guo SJ, Pei XQ, Zhang YJ, Xiao YH, Li DM, Jin K, Bidochka MJ, Pei Y (2007) Increased insect virulence in Beauveria bassiana strains overexpressing an engineered chitinase. Applied and Environmental Microbiology 73: 295-302.

Fan YH, X Pei, S Guo, Zhang YJ, Luo Z, Liao X, Pei Y (2010) Increased virulence using engineered protease-chitin binding domain hybrid expressed in the entomopathogenic fungus Beauveria bassiana. Microbial Pathogenesis 49: 376-380.

Fang W, Feng J, Fan Y, Zhang Y, Bidochka MJ, St. Leger RJ, Pei Y (2009) Expressing a fusion protein with protease and chitinase 
activities increases the virulence of the insect pathogen Beauveria bassiana. Journal of Invertebrate Pathology 102: 155-159.

Fang WG, Leng B, Xiao YH, Jin K, Ma JC, Fan YH, Feng J, Yang XY, Zhang YJ, Pei Y (2005) Cloning of Beauveria bassiana chitinase gene Bbchit1 and its application to improve fungal strain virulence. Applied and Environmental Microbiology 71: 363-370.

Firouzbakht H, Zibaee A, Hoda H, Sohani MM (2015) Virulence Determination of Beauveria bassiana Isolates on a Predatory Hemipteran, Andrallus spinidens Fabricius (Hemiptera: Pentatomidae). Acta Phytopathologica et Entomologica Hungarica 50(1): 115-125.

Gibson DM, Donzelli BG, Krasnoff SB, Keyhani NO (2014) Discovering the secondary metabolite potential encoded within entomopathogenic fungi. Natural Product Reports 31: 1287-1305.

Glare TR, Reay SD, Nelson TL, Moore R (2008) Beauveria caledonica is a naturally occurring pathogen of forest beetles. Mycological Research 112(3): 352-360.

Gupta R, Kumari A, Syal P, Singh Y (2015) Molecular and functional diversity of yeast and fungal lipases: their role in biotechnology and cellular physiology. Progress in Lipid Research 57: $40-54$

Hamid R, Khan MA, Ahmad M, Ahmad MM, Abdin MZ, Musarrat J, Javed S (2013) Chitinases: An update. Journal of Pharmacy \&BioAllied Sciences 5(1): 21-29. https://doi.org/10.4103/0975-7406.106559.

Hernandez CEM, Guerrero IEP, Hernandez GAG, Solis ES, Guzman JCT (2010) Catalase overexpression reduces the germination time and increases the pathogenicity of the fungus Metarhizium ansiopliae. Applied Microbiology and Biotechnology 87: 1033-1044.

Joshi N, Shera PS, Sangha KS, Sharma S (2019) Bioformulations for management of pod borer, Helicoverpa armigera (Hübner) in Mungbean (Vigna radiata L.). Journal of Biological Control 33(1): 76-79.

Joshi L, St. Leger RJ, Bidochka MJ (1995) Cloning of a cuticledegrading protease from the entomopathogenic fungus, Beauveria bassiana. FEMS Microbiology Letters 125: 211-217.

Kaur A, Joshi N (2014) Conidial production of Beauveria bassiana on agricultural products and effect of storage temperature on its formulations. African Journal of Microbiology 8(34): 3164-3170.

Kaur N, Dhawan M, Sharma I, Pati PK (2016) Interdependency of Reactive Oxygen Species generating and scavenging system in salt sensitive and salt tolerant cultivars of rice. BMC Plant Biology 16: 131 https://doi.org/10.1186/s12870-016-0824-2.

Kaur S, Kumar A, Joshi N (2017) Bioefficacy of Bacillus thuringiensis against cabbage butterfly, Pieris brassicae. Journal of Entomology and Zoology Studies 5(5): 1057-1061.

Khan S, Guo L, Shi H, Mijit M, Qiu D (2012) Bioassay and enzymatic comparison of six entomopathogenic fungal isolates for virulence or toxicity against green peach aphids Myzus persicae. African Journal of Biotechnology 11(77): 14193-203.

Kim JS, Roh JY, Choi JY, Wang Y, Shim HJ, Je YH (2010) Correlation of the aphicidal activity of BeauveriabassianaSFB205 supernatant with enzymes. Fungal Biology 114: 120-128.

Kwok LY, Schlüter D, Clayton C, Soldati D (2004) The antioxidant system in Toxoplasma gondii and the role of cytosolic catalase in defense against oxidative injury. Molecular Microbiology 51: 47-61.

Keyhani NO (2017) Lipid biology in fungal stress and virulence: Entomopathogenic fungi. Fungal Biology 122(6): 420-429.

Li DC (2006) Review of fungal chitinases. Mycopatholgia161: 345-360.

Li F, Shi HQ, Ying SH, Feng MG (2015) Distinct contributions of one $\mathrm{Fe}$ - and two $\mathrm{Cu} / \mathrm{Zn}$-cofactored superoxide dismutases to antioxidation, UV tolerance and virulence of Beauveria bassiana. Fungal Genetics and Biology 81: 160-71.

Litwin A, Nowak M, Rozalska S(2020) Entomopathogenic fungi: unconventional applications. Reviews in Environmental Science and Biotechnology19: 23-42.

Liu Q, Ying SH, Li JG, Tian CG, Feng MG (2013) Insight into the transcriptional regulation of Msn2 required for conidiation, multistress responses and virulence of two entomopathogenic fungi. Fungal Genetics and Biology 54: 42-51.

Liu SQ, Meng ZH, Yang JK, Fu YK, Zhang KQ (2007) Characterizing structural features of cuticle-degrading proteases form fungi by molecular modelling. BMC Structural Biology 7(33) https://dx.doi.org/10.1186\%2F1472-6807-7-33.

Mahmood DF, Abderrazak A, El Hadri K, Simmet T, Rouis M (2013) The thioredoxin system as a therapeutic target in human health and disease. Antioxidant Redox Signal 19:1266-1303 (2013).

Mancillas-Paredes JS, Hernandez-Sanchez H, Jaramillo-Flores ME, Garcia-Gutierrez C (2019) Proteases and Chitinases Induced 
in Beauveria bassiana during Infection by Zabrotes subfasciatus. Southwestern Entomologist 44(1): 125-137.

Matias-Montesinos R, Gonzales-Viniegra G, Rosas-Alatorre R, Loera O (2011) Relationship between virulence and enzymatic profile in the cuticle of Tenebrio molitor by 2-deoxy-D-glucoseresistant mutants of Beauveria bassiana (Bals.) Vuill. World Journal of Microbiology and Biotechnology 27: 2095-2102.

Meenu, Dhawan M, Joshi N (2020) Toxicity, biodegradation and bioremediation of triazophos (TAP): AMini-Review. Research Journal of Pharmaceutical, Biological and Chemical Sciences11(5):109-116.

Mustacich D, Powis G (2000) Thioredoxin reductase. Biochemical Journal 346: 1-8.

Oerke EC, Dehne HW (2004) Safeguarding production - losses in major crops and the role of crop protection. Crop Protection 23: 275-285.

Oliveira CM, Auad AM, Mendes SM, Frizzas MR (2014) Crop losses and the economic impact of insect pests on Brazilian agriculture. Crop Protection 56: 50-54.

Ortiz-Urquiza A, Keyhani NO, Quesada-Moraga E (2013) Culture conditions affect virulence and production of insect toxic proteins in the entomopathogenic fungus Metarhizium anisopliae. Biocontrol Science and Technology 23: 1199-1212.

Ortiz-Urquiza A, Keyhani NO (2014) Stress response signalling and virulence: insights from entomopathogenic fungi. Current Genetics 61(3): 239-49.

Oyeleye A, Normi YM (2018). Chitinase: diversity, limitations, and trends in engineering for suitable applications. Bioscience reports 38(4): BSR2018032300. https://doi.org/10.1042/BSR20180323.

Pedrini N, Ortiz-Urquiza A, Huarte-Bonnet C, Zhang S, Keyhani NO (2013) Targeting of insect epicuticular lipids by the entomopathogenic fungus Beauveria bassiana: hydrocarbon oxidation within the context of a host-pathogen interaction. Frontiers in Microbiology 4(24) https://doi.org/10.3389/fmicb.2013.00024.

Pedrini N, Ortiz-Urquiza A, Huarte-Bonnet C, Fan Y, Juarez MP, Keyhani NO (2015) Tenebrionid secretions and a fungal benzoquinone oxidoreductase form competing components of an arms race between a host and pathogen. Proceedings ofthe National Academy of Sciences of the United States of America 112 (28): 3651-3660.
Pedrini N, Juarez MP, Crespo R, de Alaniz MJT (2006) Clues on the role of Beauveriabassiana catalases in alkane degradation events. Mycologia 98: 528-534.

Pelizza SA, Eliades SA, Scorsetti AC, Cabello MN (2012) Entomopathogenic fungi from Argentina for the control of Schistocerca cancellata (Orthoptera: Acrididae) nymphs: fungal pathogenicity and enzyme activity. Biocontrol Science and Technology 22: 1119-1129.

Perinotto WMS, Golo PS, Rodrigues CJBC, Sa FA, Santi L, da Silva WOB, Junges A, Vainstein MH, Schrank A, Salles CMC, Bittencourt VREP (2014) Enzymatic activities and effects of mycovirus infection on the virulence of Metarhizium anisopliae in Rhipicephalus microplus. Veterinary Parasitology 203: 189-96.

Pinnamaneni R, Kalidas P, Sambasiva, Rao KRS (2010) Cloning and Expression of Bbchitl gene of Beauveriabassiana. The Open Entomology Journal 4:30-35.

Sanchez-Perez L, Rodriguez-Navarro S, Marin-Cruz VH, Ramos Lopez MA, Ramos AP, Barranco-Florido JE (2016) Assessment of Beauveria bassiana and Their Enzymatic Extracts against Metamasius spinolae and Cyclocephala lunulata in Laboratory. Advances in Enzyme Research 4: 98-112.

Santi L, Coutinho-Rodrigues CJB, Berger M, Klein LAS, De Souza EM, Rosa RL, Guimarães JA, Yates JR, Perinotto WMS, Bittencourt VREP, Beys-da-Silva WO (2018) Secretomic analysis of Beauveria bassiana related to cattle tick, Rhipicephalus microplus, infection. Folia Microbiology 64: 361-372.

Santi L, Beys da Silva WO, Berger M, Guimaraes JA, Schrank A, Vainstein MH (2010) Conidial surface proteins of Metarhizium anisopliae: source of activities related with toxic effects, host penetration and pathogenesis. Toxicon55: 874-880.

Sharma J, Singh A, Kumar R, Mittal A (2006) Partial purification of an alkaline protease from a new strain of Aspergillus oryzae AWT 20 and its enhanced stabilization in entrapped Ca-alginate beads. The Internet Journal of Microbiology 2:1-14.

Sharma T, Joshi N, Kalia A (2017) Scanning electron microscopic studies of Beauveria bassiana against Lipaphis erysimi Kalt. Journal of Applied and Natural Science 9(1): 461-465.

Singh B, Kaur N, Kumar P, Hallan V, Pati PK (2020) Reactive oxygen species generating and scavenging systems play critical role in conferring leaf spot disease resistance in Withania somnifera (L.) Dunal. Industrial Crops and Products 157:112889. 
Singh H, Joshi N (2020) Management of the aphid, Myzus persicae (Sulzer) and the whitefly, Bemisia tabaci (Gennadius), using biorational on capsicum under protected cultivation in India. Egyptian Journal of Biological Pest Control 30,67 https://doi.org/10.1186/s41938-020-00266-5.

Song TT, Ying SH, Feng MG (2012) High resistance of Isaria fumosorosea to carbendazim arises from the overexpression of an $\mathrm{ABC}$ transporter (ifT1) rather than tubulin mutation. Journal of Applied Microbiology 112: 175-184.

St. Leger RJ, Frank DC, Roberts DW, Staples RC (1992) Molecular cloning and regulatory analysis of the cuticle-degrading protease structural gene from the entomopathogenic fungus Metarhizium anisopliae. European Journal of Biochemistry 204: 991-1001.

St. Leger RJ, Joshi L, Bidochka M, Rizzo MJ, Roberts DW (1996) Biochemical characterization and ultrastructural localization of two extracellular trypsins produced by Metarhizium anisopliae in infected insect cuticles. Applied and Environmental Microbiology 62: 1257-64.

St. Leger RJ, Bidochka MJ, Roberts DW (1994) Isoforms of the cuticle-degrading Pr1 proteinase and production of a metalloproteinase by Metarhizium anisopliae. Archives of Biochemistry and Biophysics313: 1-7.

Thakur R, Rajak RC, Sandhu SS (2005) Biochemical and molecular characteristics of indigenous strains of the entomopathogenic fungus Beauveri abassiana of central India. Biocontrol Science and Technology 15(7): 733-44.

Thungrabeab M, Tongma S (2007) Effect of Entomopathogenic Fungi, Beauveria bassiana (Balsamo) and Metarhizium anisopliae (Metsch) on non-target insects. Current Applied Science and Technology7: 8-12.

Tong SM, Feng MG (2019) Insights into regulatory roles of MAPK-cascaded pathways in multiple stress responses and life cycles of insect and nematode mycopathogens. Applied Microbiology and Biotechnology 103: 577- 587.

Tong, SM, Feng MG (2020) Phenotypic and molecular insights into heat tolerance of formulated cells as active ingredients of fungal insecticides. Applied Microbiology and Biotechnology 104: $5711-5724$.

Urlacher VB, Lutz-Wahl S, Schmid RD (2004) Microbial P450 enzymes in biotechnology. Applied Microbiology and Biotechnology 64: 317-325.
Vincent F, Wegst G (2004) Design and Mechanical Property of Insect Cuticle. Arthropod Structure and Development 33:187-199.

Wang C, Hu G, St. Leger RJ (2005) Differential gene expression by Metarhizium anisopliae growing in root exudate and host (Mandu casexta) cuticle or hemolymph reveals mechanisms of physiological adaptation. Fungal Genetics and Biology 42(8): 704-18.

Wang J, Chen J, Hu Y, Ying SH, Feng MG (2020) Roles of six Hsp70 genes in virulence, cell wall integrity, antioxidant activity and multiple stress tolerance of Beauveria bassiana. Fungal Genetics and Biology144: 103437.

Wang ZL, Zhang LB, Ying SH, Feng MG (2013) Catalases play differentiated roles in the adaptation of a fungal entomopathogen to environmental stresses. Environmental Microbiology15: 409-418.

Xia L, Zeng Z, Ding X, Huang F (2009) The expression of a recombinant $C r y l A c$ gene with subtilisin-like protease $C D E P 2$ gene in acrystalliferous Bacillus thuringiensis by Red/ET homologous recombination. Current Microbiology 59: 386-392.

Xie XQ, Li F, Ying SH, Feng MG (2012) Additive contributions of two manganese-cored superoxide dismutases (MnSODs) to antioxidation, UV tolerance and virulence of Beauveria bassiana. PLoS One7https://doi.org/10.1371/journal.pone.0030298.

Xie XQ, Wang J, Huang BF, Ying SH, Feng MG (2010) A new manganese superoxide dismutase identified from Beauveria bassiana enhances virulence and stress tolerance when overexpressed in the fungal pathogen. Applied Microbiology and Biotechnology 86: 1543-1553.

Yao SL, Ying SH, Feng MG, Hatting JL (2010) In vitro and in vivo responses of fungal biocontrol agents to the gradient doses of UV-B and UV-A irradiation. Biocontrol 55: 413-422.

Yuan Y, Huang W, Chena K, Ling E (2020) Beauveria bassiana ribotoxin inhibits insect immunity responses to facilitate infection via host translational blockage. Developmental and Comparative Immunology106: 10360 https://doi.org/10.1016/j.dci.2019.103605.

Ying SH, Feng MG (2019) Insight into vital role of autophagy in sustaining biological control potential of fungal pathogens against pest insects and nematodes. Virulence 10: 429-437.

Zhang LB, Tang L, Ying SH, Feng MG (2016a) Distinct roles of two cytoplasmic thioredoxin reductases (Trr1/2) in the redox system involving cysteine synthesis and host infection of Beauveria bassiana. Applied Microbiology and Biotechnology100: 10363-10374.

Zhang LB, Tang L, Ying SH, Feng MG (2016b) Regulative roles of glutathione reductase and four glutaredoxins in 
glutathione redox, antioxidant activity, and iron homeostasis of Beauveria bassiana. Applied Microbiology and Biotechnology 100: 5907-591.

Zhang LB, MG Feng (2018) Antioxidant enzymes and their contributions to biological control potential of fungal insect pathogens. Applied Microbiology and Biotechnology 102: 4995-5004.

Zhang LB, Tang L, Ying SH, Feng MG (2015) Subcellular localization of six thioredoxins and their antioxidant activity and contributions to biological control potential in Beauveria bassiana. Fungal Genetics and Biology 76: 1-9.

Zhang S, Widemann E, Bernard G, Lesot A, Pinot F, Pedrini N, Keyhani NO (2012) CYP52X1, representing new cytochrome P450 subfamily, displays fatty acid hydroxylase activity and contributes to virulence and growth on insect cuticular substrates in entomopathogenic fungus Beauveria bassiana. The Journal of Biological Chemistry 287: 13477-13486.
Zhang YJ, Feng MG, Fan YH, Luo ZB, Yang XY, Wu D (2008) A cuticle-degrading protease (CDEP-1) of Beauveria bassiana enhances virulence. Biocontrol Science and Technology18: 551-563.

Zhou Q, Ying S, Chen A, Li W, Wang J (2018) In vivo transcriptomic analysis of Beauveria bassiana reveals differences in infection strategies in Galleria mellonella and Plutella xylostella. Pest Management Science 75(5): 1443-1452.

Zibaee A, Bandani AR, Talaei-Hassanlouei R, Malagoli D (2011) Cellular immune reactions of the sunn pest, Eurygaster integriceps, to the entomopathogenic fungus, Beauveria bassiana and its secondary metabolites. Journal of insect science(Online)11(1):138. https://doi.org/10.1673/031.011.13801.

Zimmermannn G (2007) Review on the safety of the entomopathogenic fungi Beauveria bassiana and Beauveria brongiartii. Biocontrol Science and Technology 17: 553-96. 